\title{
TRABAO SOCIAL Y CUIDADORES INFORMALES: ANÁLISIS DE LA SITUACIÓN ACTUAL Y PROPUESTA DE INTERVENCIÓN
}

\section{SOCIAL WORK AND INFORMAL CAREGIVERS: ANALYSIS OF THE CURRENT SITUATION AND SUGGESTED ACTIONS}

M. ${ }^{a}$ Caridad Fuentes Fernández (1) y Lourdes Moro Gutiérrez (2)

(1) Trabajadora Social. Servicios Sociales del Ayuntamiento de Salamanca (2) Universidad de Salamanca

Resumen: Los cambios sociales que afectan a la población española en las últimas décadas conllevan importantes modificaciones en los sistemas de relaciones interpersonales. A ello se añade el acelerado envejecimiento de dicha población que propicia la aparición de nuevas enfermedades y la cronificación de las existentes y por tanto el aumento de la dependencia funcional de estas personas debido al incremento en la esperanza de vida. Muchas eligen ser atendidas por un familiar que se convierte así en su cuidador. Esta labor de cuidador limita su actividad laboral, social y familiar e influye en su proyecto vital, afectando a su estado de ánimo y a su salud. Por ello, desde los Servicios Sociales es necesaria la creación y gestión de recursos y programas de intervención destinados a apoyar a los cuidadores. En el artículo recogemos la propuesta de intervención "El cuidador también tiene vida" con la que pretendemos capacitar a los cuidadores de enfermos de Alzheimer y otras demencias para asumir esta tarea en mejores condiciones físicas y psicológicas y conseguir una mejora en la calidad de vida del cuidador que a su vez tendrá efectos positivos sobre la persona a la que cuida.

Palabras clave: Cuidadores informales, Trabajo Social, Dependencia, Intervención.

\begin{abstract}
The social changes affecting the Spanish population in recent decades have led to some significant modifications in the systems of interpersonal relations. In addition, the aging of the Spanish population is resulting in the appearance of new diseases and the growing incidence of existing ones, and thus to an increase in the functional dependency levels of these individuals due to increased life expectancy. Many individuals choose to be cared for by a family member who thus becomes a caregiver. This task of caregiving limits these relatives professional, social and family life and has a significant impact on their life as a whole, affecting both their state of mind and their health. For this reason social services must create and manage resources and programs aimed at supporting caregivers. In the article we discuss the intervention "The caregiver has a life too," with which we hope to empower people who care for patients with Alzheimer's and other types of dementia, so that they can perform their task in better physical and psychological conditions, achieving a better quality of life for the caregiver, which will in turn have positive effects on the person receiving the care.
\end{abstract}

Key Words: Informal caregivers, Social Work, Dependence, Intervention.

| Recibido: 4/11/2013 | Revisado: 20/11/2013 | Aceptado:15/01/2014 | Publicado: 31/01/2014 |

Correspondencia: Lourdes Moro Gutiérrez. Doctora en Psicología por la Universidad de Salamanca. Profesora Titular de Universidad en la Facultad de Psicología. Docencia en los Grados de Trabajo Social, Terapia Ocupacional y Psicología. Universidad de Salamanca. Facultad de Psicología. Dirección Postal: Avenida de la Merced, 109-131. 37005 Salamanca. Tlfo. 923-294500 (ext. 3321). Email: moro@usal.es.

Referencia normalizada: Fuentes, M. C., y Moro, L. (2014). Trabajo Social y cuidadores informales: análisis de la situación actual y propuesta de intervención. Trabajo Social Hoy, 71, 43-62. doi:10.12960/ TSH.2014.0002. 


\section{INTRODUCCIÓN}

En las últimas décadas, la población española ha registrado grandes cambios sociales, que comportan a su vez notables modificaciones en los sistemas de relaciones interpersonales. Con la incorporación de la mujer al mercado laboral, la generalización del control de la natalidad y el aumento de la esperanza de vida, asistimos a un evidente envejecimiento de la población. En 1991 las personas mayores de 64 años representaban el 13,8 \% de la población, proporción que se elevó en 2001 hasta el 17 \% y que en 2011 creció hasta el 17,2 \% (Instituto Nacional de Estadística (INE), 1991, 2001 y 2012). Más significativo es el hecho de que, para las mismas fechas, los mayores de 80 años hayan aumentado desde el 2,8 \% hasta el 4,6 \% y el 6,1 \%, respectivamente, lo que implica un acelerado envejecimiento del propio envejecimiento. Como consecuencia de esta longevidad, surgen nuevas enfermedades y se agravan y cronifican las ya existentes, aumentando las limitaciones de las capacidades funcionales asociadas a la edad y constatándose una relación directa entre esta y la dependencia funcional.

Muchos de estos mayores prefieren vivir solos, aunque precisan ayuda para la realización de gran parte de las actividades de la vida diaria. Quieren envejecer en su domicilio, para mantener la independencia y evitar a la vez la soledad y el desarraigo social y familiar. Sin embargo, para ello necesitan apoyos externos de tipo personal y técnico que, en ocasiones, no cubren la totalidad de sus necesidades, dado que están prestados por familiares para quienes esta atención supone una fuerte carga de trabajo. En otros casos, es la persona dependiente quien se marcha al domicilio del familiar para ser atendida con mayor comodidad. En cualquier caso, este entramado social ha de protegerse porque estamos hablando de una atención natural entre miembros de la familia que prestan sus cuidados y desean seguir haciéndolo, aún con todos los inconvenientes generados.

Los procesos relacionados con la dependencia suelen ser largos, marcados por cuidados permanentes, e inciden negativamente en el estado de salud de la persona que cuida, porque sus atenciones requieren constantes esfuerzos físicos de movilización del enfermo y porque se enfrenta con frecuencia a enfermedades como la demencia senil, el Alzheimer y los trastornos de personalidad, que generan mayor estrés. Las consiguientes limitaciones en la actividad laboral, social y familiar de estos cuidadores influyen en su proyecto vital y perjudican su estado de ánimo y de salud (ver Figura 1). 


\section{Transformaciones sociales}

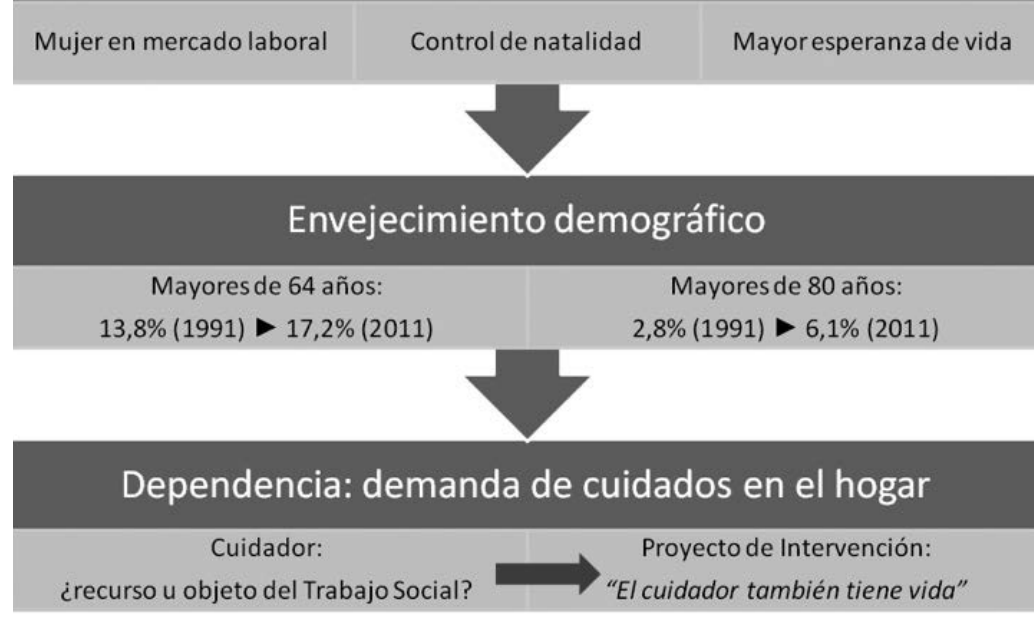

Figura 1. Justificación del Proyecto de Intervención.

Fuente: Elaboración propia.

Las políticas públicas vigentes presuponen que las familias proveen a sus miembros de los cuidados requeridos. Esto dificulta la creación y gestión de recursos destinados a ayudar a los cuidadores e impide una apropiada confluencia entre los sistemas de apoyo formal e informal que alivie la carga asociada al cuidado. Las propuestas actuales continúan tratando a las personas cuidadoras como recursos en sí mismas y no como potenciales demandantes de actuaciones preventivas o de intervención. Garantizar cuidados al cuidador es la mejor manera de que este realice su tarea durante más tiempo, retrase los procesos de institucionalización de su familiar y disfrute de mejor calidad de vida. Este trabajo presenta una propuesta de intervención que incide sobre este problema.

\section{FUNDAMENTOS TEÓRICOS}

En el marco teórico, analizamos en primer lugar la normativa vinculada al fenómeno de la dependencia, para definir después el concepto de cuidado informal. A continuación señalamos los riesgos que afectan a los cuidadores y la evaluación que precisan como requisito previo a la intervención. Finalizamos esta fundamentación presentando algunos de los recursos formales disponibles para las personas dependientes que revierten en la intervención con cuidadores (ver Figura 2). 


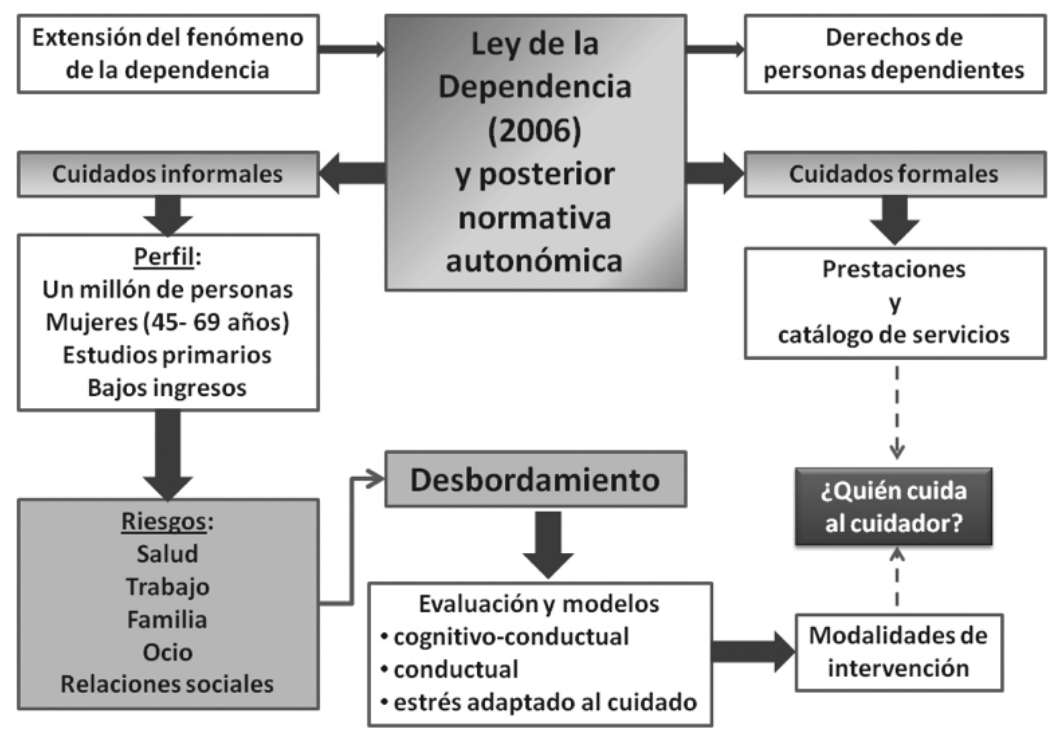

Figura 2. Fundamentación teórica.

Fuente: Elaboración propia.

\subsection{EL FENÓMENO DE LA DEPENDENCIA Y LOS CUIDADOS INFORMALES}

El Libro Blanco de la Dependencia (IMSERSO, 2005b) atribuye el incremento de la demanda de cuidados a tres factores: el envejecimiento de la población, las mayores tasas de supervivencia de las personas que padecen enfermedades congénitas, accidentes graves o patologías crónicas, y la crisis de los modelos tradicionales de apoyo informal.

Este contexto justificó la promulgación de la Ley 39/2006, de Promoción de la Autonomía Personal y Atención a las Personas en situación de Dependencia, fundamental en la determinación de los niveles de cobertura que los servicios públicos deben ofrecer a las personas dependientes. Esta ley reconoce el nuevo derecho de las personas mayores, o con alguna discapacidad que limita su autonomía y no pueden valerse por sí mismas, a recibir atenciones por parte del Estado (Caballer, Ramiro y Vivas, 2009).

Esta Ley ha sufrido posteriormente importantes modificaciones, especialmente durante 2011, con el Real Decreto 175/2011 sobre criterios para determinar las intensidades de protección de los servicios y la cuantía de las prestaciones económicas reguladas en la Ley 39/2006 y que también modifica el Real Decreto 615/2007, que regula la Seguridad Social de los cuidadores de las personas en situación de dependencia. Al- 
gunos de los aspectos más significativos que se modifican son: reducción a tres de los grados de dependencia, reducción de un $15 \%$ en la prestación por cuidador familiar y cambios en la cotización a la Seguridad Social. También se ha incluido a personas con necesidades especiales en el denominado copago farmacéutico. Por otra parte, el Real Decreto 174/2011 aprueba un nuevo baremo de valoración de la situación de dependencia. A esto se añaden las variaciones aprobadas en cada Comunidad Autónoma. Por ejemplo, Castilla-La Mancha (Ley 9/2012 de Tasas y Precios Públicos de Castilla-La Mancha y otras medidas Tributarias) ha impuesto una serie de tasas que se cobrarán al ciudadano por ciertos trámites del sistema de dependencia: cuarenta euros en caso de discrepar con la valoración realizada y solicitar una revisión, quince euros en caso de discrepar con la cantidad concedida y solicitar una revisión, o treinta euros por pedir la revisión del grado de discapacidad, cuando es evidente que los dependientes no suelen mejorar, sino que empeoran y a veces rápidamente, por lo que no es injustificada esta revisión.

Según la Encuesta sobre Discapacidad, Autonomía Personal y Situaciones de Dependencia (INE, 2008), el número de personas con discapacidad era de 3847 900, de los cuales 2,30 millones eran mujeres y 1,55 millones eran hombres. En 3,3 millones de hogares reside una persona con discapacidad y en 608000 de ellos la persona vive sola, aunque la situación más frecuente es el hogar de dos miembros donde uno de ellos presenta alguna discapacidad. Delicado, Copete, Barnés y García (2011) ratifican el perfil de las personas atendidas, al identificar a las mujeres pensionistas, mayores de 80 años y con patologías cronodegenerativas como el núcleo principal de las personas objeto de cuidados informales en el hogar.

Por tanto, y conforme planteábamos en la introducción, el cuidador doméstico debe recibir especial atención si se pretende abordar el problema de la dependencia de forma comprensiva. La Ley 39/2006, en el artículo 2.5, define los cuidados no profesionales como "la atención prestada a personas en situación de dependencia en su domicilio, por personas de la familia o de su entorno, no vinculadas a un servicio de atención profesionalizada".

Según distintos trabajos (La Parra, 2001; Nieto, 2002; IMSERSO, 2005a; IMSERSO, 2005b), el perfil mayoritario de estas personas cuidadoras, cuyo número se estimaba en 950000 para 2010 (Fernández de Larrinoa, Martínez, Ortiz, Carracos, Solabarrieta y Gómez, 2011), es el de una mujer de entre 45 y 69 años, casada, con estudios primarios, bajo nivel de ingresos, sin empleo y cuyos cuidados son prestados por iniciativa propia durante un período de cinco a nueve años. Este perfil básico no ha variado en la última década a pesar de los avances normativos y de los cambios sociodemográficos. Las mujeres continúan ejerciendo el rol de cuidadoras, papel que se perpetúa de generación en generación como demuestra el elevado número de nietas que asumen este papel (Do Muiño, Vidal, Rodríguez, Hermida y Hervés, 2009). 
No obstante, hay diferencias entre los cuidadores en función de variables como la calidad de las relaciones familiares previas al comienzo de los cuidados, el entorno inmediato en que se prestan, las creencias o el número de cuidadores disponibles (Losada, Peñacoba, Márquez y Cigarán, 2008). También surgen diferencias dependiendo de si viven en un entorno rural o urbano, del grado en que creen necesitar ayuda, de cómo utilizan los recursos, del rechazo hacia estos o de la información que tengan sobre la enfermedad y los recursos. Peeters, Van Beek, Meerveld, Spreeuwenberg y Francke (2010) concluyen que la variable que determina claramente las necesidades del cuidador es la relación con la persona enferma. Apuntan que los cuidadores demandan asesoramiento sobre la forma de abordar los problemas de comportamiento de su familiar y sobre la trayectoria de desarrollo de la enfermedad.

El nivel educativo y económico del dependiente y su familia da lugar a diferentes prácticas en la prestación de cuidados y se plasma en distintas actitudes hacia las tareas de cuidado. Los cuidadores de más alta posición socioeconómica señalan con mayor frecuencia que el principal coste de los cuidados es el tiempo que les dedican porque va en detrimento del ocio y de las relaciones sociales (Langa y Martínez, 2011). Por tanto, cualquier medida de apoyo a cuidadores debe tener en cuenta esta desigual situación de partida en cuanto al género y la clase social (García Caliente, 2004).

Brodaty y Donkin (2009) y García, Jiménez y Vilaplana (2011), entre otros autores, coinciden en identificar a la familia como proveedora fundamental de los cuidados que reciben las personas dependientes. Otras redes de apoyo serían los compañeros de trabajo, amigos, vecinos y otras organizaciones como el voluntariado, los grupos de autoayuda o las asociaciones de afectados y familiares. Estas redes sociales pueden incidir en la salud de los cuidadores induciendo a desarrollar conductas saludables, proporcionando autoestima, apoyo emocional, ayuda material y sentido de pertenencia. Como constatan Delicado et al. (2011), las personas con más redes sociales de apoyo padecen menos estrés y síntomas depresivos y muestran mayor capacidad de afrontar situaciones difíciles. Estas redes no familiares también prestan apoyo a las personas dependientes, mejorando su bienestar y sus expectativas vitales, lo que a su vez repercute positivamente en la calidad de vida del cuidador.

\subsection{LOS RIESGOS DE LOS CUIDADORES}

Los cuidadores informales realizan tareas domésticas y ayudan al dependiente en su cuidado personal y actividades cotidianas. Emplean gran parte de su tiempo en la realización de todas estas labores, en perjuicio de sus actividades profesionales, tiempo de ocio y estado de salud (Crespo y Martínez, 2007; López, Orueta, Gómez-Caro, Sánchez, Carmona y Alonso, 2009), por lo que desarrollan estrés crónico y padecen 
más problemas psicológicos y físicos que el resto de la población, teniendo un 63 \% más de posibilidades de morir en el plazo de cuatro años (Losada, Moreno-Rodríguez, Cigarán, Peñacoba y Montorio, 2006).

Losada et al. (2008) añaden posteriormente otras consecuencias negativas del cuidado, como los problemas personales en la relaciones de pareja o de amistad. Argumentan que los cuidadores tienen menos comportamientos relacionados con la salud, menores sentimientos de autoeficacia, peor estado de salud, mayores niveles de las hormonas relacionadas con el estrés y mayor riesgo de sufrir infecciones, a la vez que manifiestan más irritabilidad y mayor sintomatología depresiva. El cuidador puede optar por conductas poco saludables como no descansar lo necesario o rechazar actividades de ocio.

En suma, las personas cuidadoras están sometidas al peligro de desbordamiento y agotamiento de sus recursos personales que incrementa el riesgo de padecer diversos problemas físicos, así como importantes alteraciones emocionales (Bonafonte, 2009: p. 114), especialmente una incidencia significativa de depresión (Vázquez, Torres, Otero, Hermida y Blanco, 2012). El hecho de que los cuidadores no reciban un salario por sus cuidados no significa que los presten gratuitamente, pues un $62 \%$ señalan que su salud se ha deteriorado, un $50 \%$ considera que su vida laboral también se ve afectada y 7 de cada 10 afirman que su ocio, tiempo libre o vida familiar se han resentido (García et al., 2011), siendo especialmente estresante el cuidado de personas con demencia.

\subsection{LA EVALUACIÓN DE LOS CUIDADORES COMO REQUISITO PARA LA INTERVENCIÓN}

La evaluación inicial de los cuidadores permitirá decidir cuál es la intervención posterior más conveniente. La Family Caregiver Alliance publicó las conclusiones de varios encuentros científicos sobre la evaluación de los cuidadores (Canga, Vivar y Naval, 2011) y recomienda que esta se haga desde la perspectiva de la familia, como unión formada por la persona cuidada, la cuidadora y los demás miembros de la unidad familiar. Dicha evaluación debería desembocar en un plan de intervención multidimensional elaborado conjuntamente con el cuidador, cuyos objetivos habrían de ser concretos, evaluables y actualizables.

Existen distintos modelos teóricos de evaluación de cuidadores:

A. Modelo cognitivo-conductual (Losada, Knight, y Márquez, 2003). Pretende identificar pensamientos y creencias disfuncionales que obstaculizan la adaptación a la situación de cuidado e influyen negativamente en la forma de afrontar las emociones.

B. Modelo conductual o modelo ABC (Teri et al., 1998). Propone evaluar variables relativas a la frecuencia e intensidad de los comportamientos problemáticos asociados a la demencia y a la reacción de estrés del cuidador vinculada a es- 
tos comportamientos. Modificando las condiciones antecedentes, disminuirá la probabilidad de aparición de estos comportamientos problemáticos y mejorará el clima de cuidado. Se ha observado una forma de interactuar del cuidador con la persona dependiente en la que es frecuente que las conductas independientes sean ignoradas y los comportamientos problemáticos reforzados.

C. Modelo de estrés adaptado al cuidado. Losada et al. (2008), basándose en las aportaciones de William Haley, Leonard Pearlin y Bob Knight, proponen un modelo multidimensional según el cual para comprender el malestar psicológico y físico del cuidador hay que considerar el estrés como un proceso donde influyen diversos factores:

- Contextuales: variables sociodemográficas, personales (parentesco con la persona cuidada y relación que mantenía con ella antes del cuidado) y culturales (valores predominantes de una sociedad).

- Estresores primarios: situaciones asociadas al cuidado que demandan una respuesta por parte del cuidador y que han surgido como consecuencia de la situación del cuidado.

- Estresores secundarios: los cambios producidos en la vida de los cuidadores como consecuencia de la situación de cuidado.

- Recursos del cuidador: variables que median en la relación entre los estresores y los resultados del proceso (valoración del estrés, grado en que la persona cuidadora interpreta el cuidado como estresante, estrategias de afrontamiento y habilidades que tiene para el cuidado).

- Consecuencias: efectos que tiene el proceso de estrés en el funcionamiento psicosocial y físico en relación con el cuidado.

El conocimiento previo de estas variables y de sus interrelaciones es el paso previo para desarrollar intervenciones adecuadas a cada situación. Pinquart y Sörensen (2006) clasifican las intervenciones con cuidadores en los siguientes tipos: psicoeducativas, psicoterapéuticas, autoayuda, apoyo mutuo, programas de respiro, formativas, de consejo y gestión de casos y multidisciplinares.

En conjunto, se posee escasa evidencia de los resultados obtenidos con estos modelos porque no se ha realizado una valoración científica de la eficacia de las intervenciones (Torres, Ballesteros y Sánchez, 2008). Según Losada et al. (2008), las revisiones sistemáticas de estas intervenciones muestran que las que obtienen mejores resultados son las psicoeducativas y las psicoterapéuticas. López y Crespo (2007) señalan que, para evitar abandonos, las intervenciones deben adecuarse a las necesidades y características de las personas cuidadoras, principalmente a su disponibilidad de tiempo y a la acumulación de tareas, ofertando programas breves, que no añadan estrés ni sobrecarguen más al cuidador. 
En España, la Ley 39/2006, en sus artículos 13 a 25, regula las prestaciones y el catálogo de servicios del sistema para la autonomía y atención a la dependencia, reconociendo como tales los de prevención y promoción de la autonomía personal, teleasistencia, ayuda a domicilio, centro de día, centro de noche y atención residencial. Respecto a las prestaciones económicas, incluye las de asistencia personal, las vinculadas a alguno de los servicios citados y las asignadas para cuidados en el entorno familiar. En otros países, sin embargo, la ayuda a los cuidadores no solo se centra en prestaciones económicas o en servicios para el dependiente. En Francia y Alemania se ofrecen, respectivamente, tres y seis meses de baja laboral para cuidados, sin perder derechos. En Dinamarca, cuando una persona cambia de domicilio para atender a una persona dependiente, el Ayuntamiento debe proporcionarle otro empleo. En Grecia, los cónyuges de personas dependientes pueden jubilarse anticipadamente o con menos años de cotización. En Finlandia, los cuidadores tienen derecho a tres días de descanso al mes (García et al., 2011).

Concluimos con Fernández et al. (2011) que los servicios comunitarios no deberían orientarse únicamente a las personas dependientes, sino que también deberían centrarse en las personas cuidadoras. Es necesario poner en marcha programas multidisciplinares que den soporte a los cuidadores porque las propuestas actuales continúan tratándolos como recursos y no como pacientes (López et al., 2009; Brodaty y Donkin, 2009).

\section{3. «EL CUIDADOR TAMBIÉN TIENE VIDA»: PROPUESTA DE PROYECTO DE INTERVENCIÓN}

Enlazando con el planteamiento teórico, presentamos a continuación el diseño de nuestra propuesta de intervención. Como hemos señalado, numerosos autores y la práctica profesional del Trabajador Social, entre otros técnicos, ponen de manifiesto la necesidad de considerar al cuidador como un paciente y no solo como un recurso. Somos conscientes de la existencia de diversos protocolos de intervención de las instituciones públicas que abordan desde distintas perspectivas los problemas de los cuidadores. Sin embargo, nuestro trabajo cotidiano en Servicios Sociales pone de manifiesto que los problemas continúan sin resolverse por lo que creemos necesario concebir propuestas de actuación para los cuidadores que puedan llegar a ser más eficaces (ver Figura 3). 


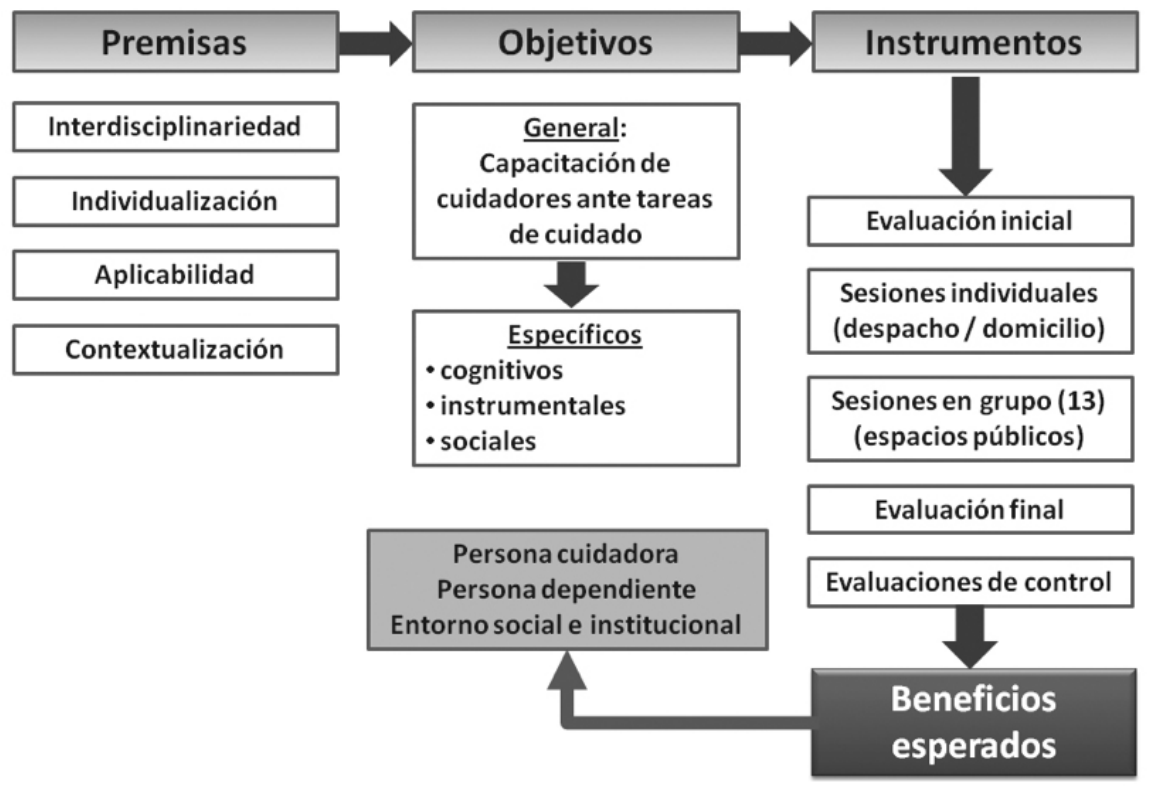

Figura 3. "El cuidador también tiene vida". Proyecto de Intervención.

Fuente: Elaboración propia.

\subsection{OBJETIVOS}

Como objetivo general, pretendemos capacitar a las personas cuidadoras de enfermos de Alzheimer y otras demencias para asumir el cuidado de su familiar en mejores condiciones físicas y psicológicas, aprendiendo a reducir los niveles de carga y malestar que genera el cuidado. El fin último de nuestro proyecto es contribuir a mejorar la calidad de vida del cuidador, lo que a su vez tendrá efectos positivos sobre la persona a la que cuida.

Objetivos específicos:

- $\quad$ Dotar de conocimientos nuevos sobre la enfermedad, sus cuidados y las maneras de afrontar las situaciones de crisis.

- $\quad$ Aprender técnicas de relajación, estrategias de afrontamiento para controlar situaciones disfuncionales y posturas idóneas para la movilización de la persona dependiente que permitan aumentar la calidad de los cuidados y dotar de recursos al cuidador para afrontar el cuidado en mejores condiciones físicas y psicológicas.

- Trabajar en el entorno de la persona dependiente y de su cuidador buscando vías que permitan compatibilizar la vida personal y profesional del cuidador con el cuidado de la persona dependiente. 


\subsection{DESTINATARIOS}

Serán las personas cuidadoras que puedan padecer problemas físicos y psicológicos derivados del papel de cuidador. Las personas susceptibles de formar parte del programa podrán venir derivadas desde los centros de salud, las asociaciones de afectados y los Centros de Acción Social. Los grupos no podrán estar integrados por más de ocho cuidadores.

\subsection{DISEÑO METODOLÓGICO}

Establece tres modalidades de interacción entre profesionales y cuidadores:

A) Evaluación inicial de los cuidadores mediante entrevistas semiestructuradas.

B) Sesiones individualizadas de intervención. Estas sesiones no tienen una duración y frecuencia previamente establecidas.

Las modalidades A y B serán responsabilidad del trabajador social que coordina el programa, aunque puede necesitar la colaboración de otros profesionales.

C) Sesiones de grupo. Estarán dirigidas por dos profesionales: un trabajador social y otro profesional que variará en función del tema y las actividades planteadas (psicólogo, fisioterapeuta, terapeuta ocupacional, abogado y neurólogo).

Al objeto de no saturar al cuidador, pero dando a la vez continuidad al programa, lo más adecuado sería realizar una sesión cada dos semanas, con una duración aproximada de dos horas, hasta completar trece intervenciones.

Las intervenciones se desarrollarán en dos espacios: (a) espacios de las instituciones públicas (centros municipales, Centros de Referencia Estatal de Atención a personas con enfermedad de Alzheimer y otras demencias, asociaciones de familiares) y (b) el domicilio de la persona cuidada.

\subsection{ACCIONES Y MEDIDAS}

Se llevarán a cabo trece sesiones, que serán explicadas a continuación.

\section{- Primera Sesión: Presentación del Programa}

En esta toma de contacto, se explicará a los cuidadores la estructura del programa, las normas de funcionamiento, lo que se espera de ellos y qué puede aportarles el programa. Se indicará que deben asistir al mayor número de sesiones posibles y la importancia de que realicen las tareas que se asignan para casa. 
Los objetivos y contenidos son los siguientes:

- Presentación del programa y breve introducción de las sesiones.

- Presentación de los profesionales.

- $\quad$ Presentación de los participantes: cada uno de ellos dirá quién es, a qué persona cuida, qué dificultades tiene a la hora de cuidarla y cómo se siente.

- Descripción de las normas de funcionamiento.

- Exposición de las expectativas de cada uno ante el programa.

Profesionales: Trabajador social. Sería interesante que pudieran participar todos los profesionales que van a colaborar, para ofrecer una visión global del programa

\section{- Segunda sesión: La importancia del cuidado del cuidador}

Esta sesión pretende abrir el debate sobre la necesidad de cuidarse y de evaluar las creencias sobre el cuidado, los obstáculos y las barreras de tipo cognitivo que impiden tomar conciencia de la necesidad de cuidarse. La manifestación continua de agotamiento y estrés es un signo evidente de que el cuidador no se encuentra bien y requiere ayuda. Objetivos y contenido de la sesión:

- Resaltar la necesidad de cuidarse y de modificar las barreras de tipo cognitivo que impiden darse cuenta de la necesidad de cuidarse.

- Motivar hacia el propio cuidado.

- Atender a signos de alarma en el cuidador como problemas con el sueño, aislamiento, consumo excesivo de medicamentos, irritabilidad, trastornos del apetito o problemas de memoria.

- Aportar pautas para el cuidado del cuidador.

- Proporcionar una plantilla de ejercicios físicos de estiramiento, tonificación, relajación y posturales, para prevenir y minimizar la sobrecarga física del cuidador.

Profesionales: Trabajador social y psicólogo.

\section{- Tercera sesión: Planificación del tiempo del cuidador}

Cuidar de una persona con Alzheimer implica realizar distintas actividades, que deben compaginarse con las que el cuidador ya realizaba. La persona cuidadora puede verse abrumada por la cantidad de actividades y no saber cómo planificar su tiempo. Una planificación realista, adaptando las actividades de forma flexible al tiempo disponible e incluyendo momentos para la realización de tareas placenteras para el cuidador, influirá positivamente en la percepción que este tiene del cuidado. Los objetivos y contenidos serán: 
- Enseñar criterios de planificación y organización de las actividades diarias, incluyendo todos los aspectos de la vida y reduciendo el malestar que provoca la falta de tiempo.

- Transmitir los beneficios que proporciona la búsqueda de ayuda para la realización de algunas tareas. Aprender a pedir ayuda cuando se necesite y a delegar tareas.

- Entrenar técnicas de comunicación asertiva para pedir ayuda y para la promoción de relaciones personales.

Profesionales: Trabajador social y psicólogo.

\section{- Cuarta sesión: Normas y sentimientos de culpa}

Es conveniente aprender a identificar las normas y reglas del cuidador en relación al cuidado de su familiar y a su propio cuidado y buscar el equilibrio entre ambas. En la sesión se presentan razonamientos para diferenciar entre las reglas más adaptativas y las que no lo son. Cuando las normas no son adecuadas, se puede tratar de hacerlas más flexibles para no sentirse mal en caso de no poder cumplirlas en alguna ocasión y así minimizar la sensación de malestar. Los objetivos y contenidos de la sesión serán:

- Identificar las normas y reglas de cada persona en los ámbitos del cuidado personal y de su familiar.

- Reflexionar sobre la relación existente entre las normas y las pautas marcadas por la sociedad.

- Diferenciar entre normas y reglas adecuadas y las que influyen negativamente en el malestar del cuidador.

- Trabajar criterios de rigidez y flexibilidad en relación con las normas y reglas. Analizar sentimientos de culpa.

- Conocer los derechos del cuidador.

Profesionales: Trabajador social y psicólogo.

\section{- Quinta sesión: Tiempo libre y realización de tareas agradables}

En esta sesión se trabaja la importancia de que el cuidador tenga tiempo libre y este sea convenientemente aprovechado. Los objetivos y contenidos de la sesión serán:

- Fomentar el tiempo libre y la realización de tareas agradables del cuidador.

- Promocionar las relaciones sociales y el tiempo de ocio y personal como cuidados para el cuidador.

- Trabajar la vinculación entre las relaciones sociales y el estado emocional.

- Aportar consejos prácticos para cuidar mejor de sí mismo.

Profesionales: Trabajador social y terapeuta ocupacional. 


\section{- Sexta sesión: Pensamientos disfuncionales}

Los sentimientos de las personas cuidadoras no dependen solamente de las situaciones que afrontan a diario, sino también de su interpretación de la realidad y de sus pensamientos sobre las situaciones que tienen que vivir. Los pensamientos negativos son prácticas adquiridas, así que pueden y deben ser identificados para diferenciar entre los que provocan emociones positivas y aquellos que les hacen sentir mal. Los objetivos y contenidos de la sesión serán:

- Trabajar el manejo de diferentes estrategias para reducir el malestar generado por los pensamientos disfuncionales y desadaptativos.

- Explicar la relación entre situación, pensamiento y emoción.

Profesionales: Trabajador social y psicólogo.

\section{- Séptima sesión: Manejo de la frustración o enfado}

La frustración y el enfado son síntomas comunes en los cuidadores de personas con Alzheimer. Pueden estar provocados por: desconocimiento de la enfermedad, pensamientos poco realistas o disfuncionales sobre cómo debe cuidar o por la falta de habilidades para afrontar los pensamientos negativos y para comunicarse. Se enseñarán técnicas de comunicación asertiva y se promoverá el conocimiento de las causas que provocan tensión. Los objetivos y contenidos de la sesión serán:

- Entrenar habilidades para el manejo de la frustración o enfado en los cuidadores.

- Conocer y analizar las causas que pueden generar frustración o ira.

- Explicación del modelo cognitivo-conductual: importancia de los pensamientos positivos y de la realización de actividades agradables.

Profesionales: Trabajador social y psicólogo.

\section{- Octava sesión: Características de las demencias. La enfermedad de Alzheimer}

Esta sesión pretende poner una base para la comprensión de la enfermedad, combatiendo algunas ideas que puedan tener los cuidadores en relación, por ejemplo, con conductas no deseadas de sus familiares y que les suponen una mayor carga de estrés y una fuente de conflictos, como pensar que sus familiares intentan fastidiarles o están aburridos por el hecho de que pueden pasar buena parte del día persiguiéndoles. La sesión se desarrolla en este momento, y no al principio de la intervención grupal como cabría esperar, porque el objetivo del programa se centra sobre todo en el bienestar del cuidador. Los objetivos y contenidos de la sesión serán: 
- Trabajar el concepto de demencia y específicamente de la demencia tipo Alzheimer.

- Proporcionar conocimientos sobre las características de la enfermedad y su evolución: fases, cronicidad y brotes.

- Reconocer conductas que se asocian a la enfermedad y que son responsables de la mayor sobrecarga de las personas cuidadoras, como la agresividad, el vagabundeo o la agitación.

- Conocer las deficiencias más frecuentes en las distintas áreas:

- Cognitiva: pérdida de memoria, problemas de lenguaje, desorientación, praxias, gnosias, alteraciones en el razonamiento y en el cálculo.

- Motora: conocer las posibles afectaciones en las tres fases: autónoma, dependencia parcial y dependencia total.

- Conductual y psicológica: alteraciones del estado de ánimo, aplanamiento afectivo, alteraciones conductuales como desvestirse en público o gritar, trastornos neurovegetativos y disfagia.

Profesionales: Trabajador social y neurólogo.

\section{- Novena sesión: Conductas no deseadas}

Se analizará brevemente la teoría de Linda Teri sobre la reducción de comportamientos problemáticos. Según la autora, estos comportamientos se mantienen porque los suscitan determinadas situaciones antecedentes o bien porque, tras la conducta no deseada, ciertas situaciones provocan que dicha conducta se repita.

Se explicarán estrategias para prevenir o manejar mejor las conductas conflictivas de la persona cuidada. Se insistirá en que muchos de los inconvenientes que surgen en el cuidado de un enfermo de Alzheimer son debidos a la falta de comunicación por los problemas de comprensión del afectado. Los objetivos y contenidos de la sesión serán:

- Trabajar el modelo ABC de los comportamientos problemáticos (antecedente, comportamiento y consecuentes).

- Dar pautas de actuación para minimizar las conductas no deseadas en la persona cuidada e identificar los comportamientos problemáticos que tratar: depresión, agresividad, agitación, insomnio, ansiedad, vagabundeo, acusaciones injustas, demandas excesivas, manifestaciones psicóticas, conductas repetitivas y ruidosas.

- Trabajar la comunicación con la persona cuidada:

- Comportamientos comunicativos como antecedentes de conductas problemáticas.

- Comunicación verbal y no verbal.

- Estrategias para una comunicación efectiva.

Profesionales: Trabajador social y psicólogo. 


\section{- Décima sesión: Cuidados a la persona dependiente. Promoción de la autonomía}

Durante esta sesión se darán pautas para que el cuidador aprenda la mejor forma de vestir, asear, mover y proporcionar cuidados posturales a la persona dependiente, intentando facilitar su tarea a la vez que se proporciona seguridad a la persona cuidada. También se practicarán habilidades para acompañamiento en la marcha. Los objetivos y contenidos de la sesión serán:

- Informar sobre técnicas de movilización y acompañamiento para minimizar los riesgos de salud de los cuidadores derivados de la movilización de personas dependientes y garantizar la seguridad en estas últimas.

- Promover la autonomía de la persona dependiente.

- Trabajar técnicas de acompañamiento en la marcha: marcha guiada, marcha acompañada con bastón, marcha con andador, marcha acompañada de cara.

- Especificar técnicas para acompañamiento en silla de ruedas.

- Enseñar cuidados posturales, prevención de úlceras y cuidados de la piel.

- Plantear orientaciones prácticas para realizar las tareas de higiene y vestido.

Profesionales: Trabajador social, fisioterapeuta y terapeuta ocupacional.

\section{- Undécima sesión: Intervención en el entorno inmediato de la persona dependiente}

Las medidas de adaptación de la vivienda sería preferible adoptarlas cuando el enfermo tiene todavía capacidad de aprendizaje e ir introduciendo modificaciones a medida que las vaya necesitando. Las propuestas de remodelación de las distintas estancias de la casa son múltiples, por lo que, coincidiendo con esta sesión, se realizará una visita al domicilio para determinar las modificaciones y ayudas técnicas más adecuadas y explicar al cuidador la forma de aplicarlas. Siempre que se necesitara y fuera solicitado, se podrían realizar con la familia compras guiadas de las ayudas técnicas requeridas.

Los objetivos y contenidos de la sesión serán:

- Informar y asesorar sobre las posibilidades de modificación del entorno inmediato para incrementar la autonomía en las actividades básicas e instrumentales de la vida diaria de la persona dependiente.

- Enseñar la supresión de barreras arquitectónicas y de comunicación. Dar información para la adaptación de los edificios y las viviendas.

- Mostar ayudas técnicas para la realización de adaptaciones en el entorno inmediato que favorezcan la realización de actividades de la vida diaria. Nuevas tecnologías y domótica.

- Prevención de accidentes.

Profesionales: Trabajador social y terapeuta ocupacional. 


\section{- Duodécima sesión: Apoyos formales e informales}

Los costes económicos del cuidado a personas con Alzheimer son elevados, por lo que se informará a los asistentes de los recursos formales existentes, de la solicitud de valoración de la situación de dependencia de su familiar y del acceso a las prestaciones. Se trata de mostrar que la ayuda a domicilio o los centros de día suponen un respiro para el cuidador y de salvar el miedo a usarlos. Los objetivos y contenidos de la sesión serán:

- Informar de las prestaciones y catálogo de servicios proporcionados por la Ley 39/2006 y forma de acceso a ellos:

- Servicios de prevención y promoción de la autonomía personal, teleasistencia, ayuda a domicilio, centro de día, centro de noche y atención residencial.

- Prestaciones económicas para cuidados en el entorno familiar, asistencia personal y prestación económica vinculada.

- Alentar el uso de servicios de respiro como ayuda al cuidador.

- Plantear el papel de los grupos de autoayuda y el asociacionismo.

- Informar de las ayudas en el entorno: transporte especial, servicios de asistencia en tren, avión y barco, adaptación del coche privado, utilización de autobuses adaptados y eurotaxi.

Profesionales: Dos trabajadores sociales.

\section{- Decimotercera sesión: Información jurídica. Aspectos legales de la enfermedad}

La enfermedad de Alzheimer se caracteriza por la progresiva pérdida de las facultades mentales, hecho que puede plantear implicaciones legales que pongan a los cuidadores en posición de tomar decisiones que no puedan asumir. A menos que la persona haya tomado medidas previas a la enfermedad para tratar estas situaciones, sus familiares se pueden ver abocados a resolverlas cuando la persona ya no tiene capacidad de decidir. Los objetivos y contenidos de la sesión serán:

- Información jurídica y aspectos legales de la enfermedad: informar sobre las variantes de legalización de la situación de la persona con demencia y cómo llevarlas a cabo.

- Conocer las posibles figuras legales: tutela, curatela y guarda legal.

- Analizar los deberes de los tutores legales.

Profesionales: Trabajador social y abogado. 


\subsection{MECANISMOS DE EVALUACIÓN}

La evaluación tendrá cuatro momentos diferenciados: evaluación previa de los cuidadores, continua, final al terminar el programa y de control tras la finalización de la intervención.

El Alzheimer supone una lesión en el cerebro del paciente y una estocada en la vida del cuidador, por lo que consideramos necesario que los Servicios Sociales apoyen a las personas cuidadoras en el cumplimiento de su tarea en las mejores condiciones posibles para sí mismas y para sus familiares.

\section{CONCLUSIÓN}

Con este artículo hemos pretendido explicar las trece sesiones de una intervención multidisciplinar que pretende capacitar a los cuidadores de enfermos de Alzheimer y otras demencias para asumir esta tarea en mejores condiciones físicas y psicológicas y conseguir una mejora en la calidad de vida del cuidador que a su vez tendrá efectos positivos sobre la persona a la que cuida. Esperamos que esta iniciativa pueda resultar de utilidad para los profesionales que decidan abordar este tipo de acciones.

\section{REFERENCIAS BIBLIOGRÁFICAS}

Bonafonte, F. (2009). Familia y dependencia: una mirada distinta, una respuesta diferente. En R. Sánchez Ordóñez (Coord.), Psicología y Dependencia. De la ley a la intervención psicosocial. Salamanca: Publicaciones Universidad Pontificia de Salamanca.

Brodaty, H. y Donkin, M. (2009). Family caregivers of people with dementia. Dialogues in Clinical Neuroscience, 11 (2), 217-228.

Caballer, V. (Ed.); Ramiro, P. y Vivas, D. (Coords.). (2009). Valoración de la dependencia, Madrid: Pirámide.

Canga, A.; Vivar, C.; y Naval, C. (2011). Dependencia y familia cuidadora: reflexiones para un abordaje familiar. Anales del Sistema Sanitario de Navarra, 34 (3), 463-469.

Crespo, M., y López Martínez, J. (2007). El estrés en cuidadores mayores dependientes. Madrid: Pirámide.

Delicado, M. ${ }^{a}$ V.; Copete, M. ${ }^{a}$ T.; Barnés, A.; y García, S. (2011). Redes sociales y recursos de apoyo a cuidadores familiares y personas dependientes: Comparación 2001-2009. Portularia, 2(XI), 33-45.

Do Muiño, M.; Vidal, M.; Rodríguez, E. y Hervés, E. (2009). "Perfil sociocultural de los cuidadores de pacientes inmovilizados en Atención Primaria". Cuadernos de Atención Primaria, 16, 280-283. 
Fernández de Larrinoa, P.; Martínez, S.; Ortiz, N.; Carrasco, M.; Solabarrieta, J. y Gómez, I. (2011). Autopercepción del estado de salud en familiares cuidadores y su relación con el nivel de sobrecarga. Psicothema, 3 (23), 388-393.

García Caliente, M. (2004). El sistema informal de cuidados en clave de desigualdad. Gaceta Sanitaria, 18(1), 132-139.

García, P.; Jiménez, S. y Vilaplana, C. (2011). Cuidados no profesionales y atención a la dependencia: los cuidados informales. Papeles de Economía Española, 129, 83-97.

IMSERSO (2005a). Cuidados a las personas mayores en los hogares españoles. Madrid: Ministerio de Trabajo y Asuntos Sociales.

IMSERSO (2005b). Libro Blanco de la Dependencia. Madrid: Ministerio de Trabajo y Asuntos Sociales.

INSTITUTO NACIONAL DE ESTADÍSTICA (1991, 2001). Censo de Población y Viviendas. Madrid: INE. Recuperado de: http://www.ine.es/inebmenu/mnu_cifraspob. htm

INSTITUTO NACIONAL DE ESTADÍSTICA (2008). Encuesta sobre Discapacidad, Autonomía Personal y Situaciones de Dependencia. Madrid: INE.

INSTITUTO NACIONAL DE ESTADÍSTICA (2012). Padrón Municipal de Habitantes 2011. Madrid: INE. Recuperado de: http://www.ine.es/inebmenu/mnu_cifraspob. htm

La Parra, D. (2001). Contribución de las mujeres y los hogares más pobres a la producción de cuidados de salud informales. Gaceta Sanitaria, 15 (6), 408-505.

Langa, D., y Martínez, D. (2011). Los cuidadores de familiares dependientes en Andalucía desde una perspectiva de clase. Papeles de Economía Española, 129, 66-82.

López, J., y Crespo, M. (2007). Intervenciones con cuidadores de familias mayores dependientes: una revisión. Psicothema, 19 (1), 72-80.

López, M. a J., Orueta, R., Gómez-Caro, S., Sánchez, A., Carmona, J., y Alonso, F. J. (2009). El rol de cuidador de personas dependientes y sus repercusiones sobre su Calidad de Vida y su Salud. Revista Clínica de Medicina de Familia, 2 (7), 332-334.

Losada, A., Knight, B., y Márquez, M. (2003). Barreras cognitivas para el cuidado de personas mayores dependientes. Influencia de las variables socioculturales. Revista Española de Geriatría y Gerontología, 38(2), 116-123.

Losada, A.; Moreno-Rodríguez, R., Cigarán, M., Peñacoba, C., y Montorio, I. (2006). Análisis de programas de intervención psicosocial en cuidadores de pacientes con demencia. Informaciones Psiquiátricas, 184 (2), 173-186.

Losada, A., Peñacoba, C., Márquez, M., y Cigarán, M. (2008). Cuidar cuidándose. Evaluación e intervención interdisciplinar con cuidadores familiares de personas con demencia. Madrid: Ediciones Encuentro.

Ministerio de Asuntos Sociales (2006). Ley 39/2006, de 14 de diciembre, de Promoción de la Autonomía Personal y Atención Personal y Atención a Personas en Situación de Dependencia. Boletín Oficial del Estado n. 299 de 15/12/2006: 44 142-44 156. 
Nieto, M. (2002). Ante la enfermedad de Alzheimer. Bilbao: Desclée De Brouwer.

Peeters, J., Van Beek, A., Meerveld, J., Spreeuwenberg, P., y Francke, A. (2010). Informal caregivers of persons with dementia, their use of and needs for specific professional support: a survey of the National Dementia Programme. BCM Nursing, 9: 9. Recuperado de: http://www.biomedcentral.com/1472-6955/9/9.

Pinquart, M., y Sörensen, S. (2006). Helping caregivers of persons with dementia: Which interventions work and how large are their effects? International Psychogeriatrics, 11, 1-19.

Teri, L., Logsdon, R., Whall, A., Weiner, M., Trimmer, C., Peskind, E., y Thal, L. (1998). Treatment for Agitation in Dementia Patients: A Behavior Management Approach. Psychoterapy, 35, 436-443.

Torres, M. ${ }^{a}$ P., Ballesteros, E., y Sánchez, P. D. (2008). "Programas e intervenciones de apoyo a los cuidadores informales en España". Gerokomos, 19 (1), 9-15.

Vázquez, F., Torres, A., Otero, P.; Hermida, E., y Blanco, V. (2012). La necesidad de la prevención de la depresión en los cuidadores informales. Psicologia.com, 16:15. http://hdl.handle.net710401/5506. 